\title{
Vivências Acadêmicas e Sofrimento Psíquico de Estudantes de Psicologia
}

\author{
Antonio dos Santos Andrade \\ Universidade de São Paulo, Ribeirão Preto, Brasil. \\ Natália Amaral Antunes \\ Universidade de São Paulo, Ribeirão Preto, Brasil. \\ Pedro Alves Zanoto \\ Universidade de São Paulo, Ribeirão Preto, Brasil.
}

\author{
Gabriel Arantes Tiraboschi \\ Universidade de São Paulo, Ribeirão Preto, Brasil. \\ Paulo Vinícius Bachette Alves Viana \\ Universidade de São Paulo, Ribeirão Preto, Brasil. \\ Rafael Trebi Curilla \\ Universidade de São Paulo, Ribeirão Preto, Brasil.
}

Resumo: A vivência estudantil na universidade é uma questão pouco investigada e discutida, especialmente nos cursos de Psicologia, assim como o sofrimento que pode acompanhá-la, decorrente sobretudo do contato com o sofrimento psíquico de outras pessoas ou os conteúdos acadêmicos mais diretamente relacionados com a subjetividade humana. Este artigo apresenta dados de uma pesquisa cujo objetivo era o levantamento das vivências acadêmicas dos estudantes de Psicologia de uma universidade pública do interior paulista. Na coleta de dados foi utilizado um instrumento, contendo uma questão de identificação sociodemográfica, um conjunto de 20 itens de múltipla escolha (formulado como numa escala do tipo likert de cinco pontos) e cinco questões abertas, que foi respondido por 119 estudantes dos cinco anos do curso. Os resultados, após serem submetidos às análises quantitativa e qualitativa, permitiram verificar que, na Escala Likert, os alunos apresentaram, no geral, percepção favorável do curso. No entanto, nas questões abertas referentes às vivências e ao sofrimento psíquico foram apresentados indicadores explícitos de mal-estar universitário. Na última questão, que indagava sobre a vivência de sofrimento psíquico, 107 dos 119 estudantes responderam afirmativamente. A partir destes dados, se discute a necessidade urgente de ações, tanto por parte dos gestores universitários quanto das entidades que fiscalizam a formação de psicólogos.

Palavras-chave: Ensino Superior, Estudantes Universitário - Psicologia, Serviços de Saúde Mental, Orientação Escolar.

\section{Academic Experiences and Psychological Suffering among Psychology students}

\begin{abstract}
Academic experiences constitute a poorly discussed and researched subject, which is also truth for the suffering that may go along with such experiences, especially in the psychology courses, as well as the suffering that can accompany them, stemming mainly from contact with the psychic suffering of others or with an academic content more directly related to human subjectivity. This study presents data from a survey whose purpose was to map the academic experiences of psychology students, at a Brazilian public University. A questionnaire containing a question of socio-demographic identification, a set of 20 multiple-choice items (formulated as a Likert scale of five points) and five open questions was used to collect data by 119 students of the five years of the course. The results, after being subject to quantitative and qualitative analysis, allowed to verify, in Likert scale, that the students presented, in general, a favorable perception of the course. However, the open questions relating to experiences and psychological distress presented explicit indicators of university unease. In the last question, that inquired about the experience of psychological distress, 107 of 119 students responded affirmatively. From these data, we discuss the urgent need for action, both by university administrators and by the entities that oversee the training of psychologists.
\end{abstract}

Keywords: Higher Education, College Students - Psychology, Mental Health Services, School Counseling. 


\section{Vivencias Académicas y el Sufrimiento Psíquico de los estudiantes de Psicología}

Resumen: La experiencia de los estudiantes en la universidad es un asunto poco investigado y discutido, sobre todo en los cursos de psicología, así como el sufrimiento que puede acompañarla, derivados principalmente del contacto con el sufrimiento psíquico de los demás, o con contenidos académicos más directamente relacionados con la subjetividad humano. Este artículo presenta datos de una encuesta cuyo objetivo fue estudiar las experiencias académicas de los estudiantes de psicología en una universidad pública de São Paulo. Para la recolección de datos se utilizó un cuestionario que contiene una pregunta de identificación sociodemográfico, un conjunto de 20 preguntas de elección múltiple (formulado como una escala Likert de cinco puntos) y cinco preguntas abiertas que fueron respondidas por 119 estudiantes de los cinco años del curso. Los resultados, después de haber sido objeto de un análisis cuantitativo y cualitativo, permiten verificar, en escala Likert, que los estudiantes presentan, en general, una percepción favorable del curso. Sin embargo, en las preguntas abiertas, relacionadas con las experiencias y los trastornos psicológicos, se presentaron indicadores explícitos de malestar universitario. En la última pregunta, que trataba acerca de la experiencia de sufrimiento psíquico, $107 \mathrm{de}$ 119 estudiantes respondieron afirmativamente. A partir de estos datos, se discute la necesidad urgente de adoptar medidas, tanto por parte de los administradores de la universidad, como por parte las entidades que supervisan la formación de los psicólogos.

Palabras clave: Educacion Superior, Estudiantes Universitarios (Psicologia), Servicios de Salud Mental, Orientación Escolar.

\section{Introdução}

As universidades públicas paulistas atravessam a pior crise de sua história, com gastos com pessoal muito acima do permitido pela legislação. As universidades federais não se encontram em situação mais favorável. Nestes tempos, em que cortes e contingenciamentos, demissão voluntária para servidores técnicos e administrativos, terceirização do setor administrativo, avaliação de desempenho com vistas a modificações nos regimes de trabalho ou até mesmo a exoneração de docentes são as palavras de ordem na gestão destas universidades, $o$ atendimento ao aluno em suas necessidades fundamentais é deixado em último plano.

O presente artigo pretende chamar a atenção para essa necessidade, mostrando, a partir de uma pesquisa realizada com 119 alunos do curso de Psicologia de uma universidade pública do interior paulista, a demanda por serviços de atendimento psicológico com vistas a minimizar o sofrimento psíquico destes alunos. Destaca a gravidade da questão que, a despeito da justificativa de situação de crise, não pode ser descuidada ou ter as providências a respeito adiadas.

A formação em Psicologia, em nosso país, é regulamentada pela Resolução da Câmara de Ensino Superior (CES) do Conselho Nacional de Educação
(CNES) no 05, de 15 de março de 2011, que institui as Diretrizes Curriculares Nacionais. Elaborada a partir de ampla discussão, fundamenta-se nas concepções de competências e habilidades, organizando-as em um Núcleo Comum, com seus Eixos Estruturantes, e Partes Diversificadas, com suas Ênfases Curriculares. Propõe e regulamenta ainda a criação de Estágios Supervisionados de dois tipos: Básicos, no Núcleo Comum e Específicos, nas Partes Diversificadas. Finalizando, em seu penúltimo parágrafo, estabelece a exigência de previsão no Projeto do Curso de um Serviço de Psicologia, no qual os alunos possam realizar atividades de atendimento à comunidade, na forma de estágios supervisionados.

No entanto, é possível se questionar até que ponto o formato proposto por essas Diretrizes contempla a singularidade das vivências acadêmicas do estudante de Psicologia, o qual está em contato com o sofrimento psíquico de outras pessoas e com conteúdos acadêmicos diretamente relacionados com a subjetividade humana. Estudos apresentados a seguir apontam evidências de que este contato pode não apenas induzir sofrimento psíquico no próprio estudante, como também elevar as probabilidades de desenvolvimento de desfechos mais severos. 
Identificar indicadores de sofrimento psíquico de alunos de Psicologia se constituiu na meta principal da pesquisa que será relatada neste artigo. Pretende-se ainda propor a discussão e o debate sobre as responsabilidades em relação a uma situação que vem se agravando, a cada ano, de negligência e desamparo aos estudantes de Psicologia em relação às suas demandas por atendimento psicológico. Negligência que parece ser mais acentuada nas universidades públicas, pois as Instituições de Ensino Superior (IES) particulares, com os altos índices de evasão, se veem forçadas a buscar as formas de conte-la, apostando no atendimento psicoterapêutico do aluno como uma saída. Esperando-se assim contribuir para o aprofundamento deste debate, este artigo assume como problema a existência de sofrimento psíquico na formação de psicólogos sem a iniciativa por parte dos dirigentes em ofertar serviços de atendimento.

\section{Sofrimento psíquico: prevalência dos transtornos mentais menores}

Para Horta, Horta e Horta (2012), o conceito de "distúrbio psiquiátrico menor" pode ser empregado para caracterizar conjuntos de manifestações de mal-estar psíquico, de caráter inespecífico, com repercussões fisiológicas e psicológicas que podem gerar limitações. Enquanto Cerchiari, Caetano e Faccenda (2005a), acompanhando diversos outros autores, preferem referirem-se a este fenômeno como "Transtornos Mentais Menores (TMM)", e apontam que na população mundial, em geral, os índices de prevalência destes variam entre $7 \%$ e $26 \%$, enquanto na população brasileira são de $8 \%$ a $23 \%$. Nos EUA, o índice de prevalência de TMM nas universidades é de 35\%. Entre estudantes canadenses, encontrou-se um índice de $30 \%$. E foi encontrado um índice de 39,32\% entre universitários mexicanos. Enquanto na Finlândia o índice variou de $6 \%$ a $29 \%$, segundo Cerchiari et al. (2005a). No Brasil, em universidades públicas, os índices de prevalência de TMM variaram entre $25 \%$ (Cerchiari et al., 2005a) e 58\% (Neves, \& Dalgalarrondo, 2007). Nos questionários de rastreamento, os principais sintomas investigados são os níveis de estresse, distúrbios psicossomáticos, irritabilidade, fadiga e insônia, entre outros possíveis. Horta et al. (2012), em uma universidade do sul do país, encontraram uma prevalência de $9,5 \%$ entre os professores, $19,5 \%$ entre os funcionários e $26,6 \%$ entre os estudantes.
Neves e Dalgalarrondo (2007) e Cerchiari et al. (2005a) pesquisaram diversos cursos das três grandes áreas do conhecimento (humanas, exatas e biológicas) e em ambos os estudos foi encontrada prevalência maior em estudantes dos cursos de ciências humanas e da saúde. Facundes e Ludemir (2005) pesquisaram especificamente a incidência desses transtornos em estudantes da área da saúde (cursos de Medicina, Odontologia, Enfermagem e Educação Física) e encontraram um índice geral de $34,1 \%$, com predominância entre estudantes de Medicina (42,6\%). Lima, Domingues e Cerqueira (2006) e Fiorotti, Rossoni, Borges e Miranda (2010) encontraram índices de $44,6 \%$ e $37,1 \%$, respectivamente, entre os graduandos em Medicina. Cavestro e Rocha (2006) obtiveram índices parecidos de depressão e risco de suicídio entre estudantes dos cursos de Terapia Ocupacional, Medicina e Fisioterapia, de uma universidade pública.

Costa et al. (2010), ao estimarem a prevalência de TMM e fatores associados entre estudantes de Medicina da Universidade Federal de Sergipe, encontraram uma prevalência de $40 \%$, quando se retira os dados referentes aos calouros ( $1^{\circ}$ semestre), e $42,5 \%$ quando se inclui os dados referentes aos calouros. Em estudo posterior (Costa et al., 2014), com estudantes de Medicina, Odontologia e Enfermagem, encontraram uma prevalência para os mesmos transtornos de $33,7 \%$. Em um curso de Medicina da Universidade Estadual Paulista "Júlio de Mesquita Filho" (Unesp), Silva, Cerqueira e Lima (2014) encontraram uma prevalência de $44,9 \%$ para este mesmo tipo de transtorno.

O único estudo deste tipo encontrado na literatura científica nacional que inclui estudantes de Psicologia é o de Gastaud et al. (2006), no qual a prevalência dos transtornos psiquiátrico menores foi comparada com a dos alunos de Direito e Medicina (obtida em estudo anterior, citado pelos autores). Os níveis de prevalência foram aproximadamente os mesmos, pois na pesquisa anterior haviam encontrado uma prevalência de 17,5\% para os alunos de Direito e $20 \%$ para os alunos de Medicina, enquanto na pesquisa em consideração encontraram $21,1 \%$ de prevalência destes transtornos para os de Psicologia. Não apenas com os estudantes de Psicologia, mas também nos demais estudos, anteriormente citados, os autores concluíram que o contato com o sofrimento psíquico de outras pessoas ou os conteúdos acadêmicos mais diretamente relacionados com a subjetividade humana pode elevar as probabilidades do desenvolvimento de algum transtorno mental menor. 


\section{Sofrimento Psíquico e manifestações associadas: dependência química, sintomas psicossomáticos e evasão}

No Ensino Superior, segundo Xavier, Nunes e Santos (2008), observa-se a produção de situações que propiciam sofrimento psíquico e suas manifestações sintomáticas: absenteísmo, depressão, dependência química, melancolia, fobias, isolamento e, no limite, a evasão. Os autores relatam que, ao longo de quatro anos de atendimento psicoterápico do Núcleo de Atendimento e Práticas Psicológicas (NAPP) da Universidade Estadual do Ceará (UECe) aos alunos de graduação, puderam constatar consequências do sofrimento psíquico e do mal-estar discente a partir de observação qualitativa dos sujeitos atendidos que variavam desde absenteísmo laboral e stress até dependência química e quadros de doença mental.

Andrade et al. (2014) buscaram descrever os diferentes processos que interferem no sofrimento psíquico discente em todas as escolas médicas do Ceará, acompanhando, do 20 ao 60 ano, 40 alunos da UECe e $20 \%$ dos demais estudantes com ingresso comum em todas as outras escolas cearenses. Foi utilizado o Self-Report Questionnaire-20 para avaliar Transtornos Mentais Leves (TML). Na UECe foi encontrada uma prevalência de suspeitos de portar TML de 53,3\%, sendo que $20 \%$ dos alunos procuraram ajuda psicológica. Nas outras escolas, $48,5 \%$ foram suspeitos e $18,2 \%$ procuraram ajuda. Depressão, insônia, problema pessoal, privação de lazer e insegurança técnica atuaram sobre o sofrimento.

Ferri-de-Barros, Alencar, Berchielli e Castelhano Junior (2011) investigaram a ocorrência de cefaleias entre estudantes de Medicina e de Psicologia. Foi utilizado um questionário respondido pelos estudantes. A cefaleia pelo menos uma vez na vida ocorreu em 98\% dos estudantes; no ano anterior à coleta dos dados, em $91 \%$. Enquanto a cefaleia tensional ocorreu em $59 \%$ e a enxaqueca em $22 \%$ na Medicina, na Psicologia ocorreu $48,5 \%$ e $32 \%$, respectivamente. De todos os estudantes, $45 \%$ relataram interferência variável na produtividade. No geral, a taxa de automedicação foi de $77 \%$; relataram piora da cefaleia desde o ingresso na universidade $36 \%$. Os autores consideraram que a prevalência de cefaleias foi muito alta. A enxaqueca foi mais prevalente em Psicologia do que Medicina, havendo piora dos sintomas desde o ingresso na universidade.
Santos, Pereira e Siqueira (2013) buscaram traçar o perfil do uso de álcool e tabaco entre universitários do curso de graduação em Psicologia do Centro de Ciências Humanas e Naturais da Universidade Federal do Espírito Santo com uma amostra de 221 estudantes e a utilização de um questionário. Encontrou-se maior prevalência de álcool $(85,07 \%)$ e tabaco $(33,07 \%)$ na frequência uso na vida, sendo o uso de álcool maior que na população geral. As substâncias associadas ao uso de álcool foram a maconha, os tranquilizantes e as anfetaminas. Já para o uso de tabaco, as substâncias mais associadas foram maconha, inalantes, alucinógenos e as anfetaminas.

Chiapetti e Serbena (2007) investigaram o uso de álcool, tabaco e drogas por alunos da área de saúde de uma universidade particular da cidade de Curitiba. Um questionário foi aplicado a 538 estudantes, de 18 a 54 anos, dos Cursos de Educação Física, Fisioterapia, Nutrição e Psicologia. Verificaram um elevado consumo de álcool e tabaco, principalmente nos cursos de Educação Física e Psicologia, que também se destacam no consumo de outras substâncias.

Correlacionada aos problemas da saúde mental e da falta de estrutura da universidade em termos de apoio pedagógico, está a evasão universitária, que é definida por Castro (2012) como o desligamento do curso superior por qualquer outro motivo que não a diplomação. Silva Filho, Motejunas, Hipolito e Lobo (2007) apontam uma média de evasão de $12 \%$ para as universidades públicas. Outros estudos apontam que o 10 ano da universidade é o período mais delicado para o aluno; de extrema importância para o sucesso ou não do aluno, é nesse ano que muitas desistências acontecem (Marinho-Araujo, \& Bisinoto, 2011; Reason, Terenzini, \& Domingo, 2006; Teixeira, Dias, Wottrich, \& Oliveira, 2008). É importante lembrar-se da preocupação apresentada por Bardagi e Hutz (2009) em relação ao fato de muitos alunos estarem insatisfeitos com sua experiência acadêmica, em boa parte da graduação.

De uma forma geral, os estudos apresentados nessa seção apontam diversas manifestações associadas ao sofrimento psíquico, desde a dependência química e os sintomas psicossomáticos até o absenteísmo e a evasão. Considerando-se os índices superiores à população geral para os TMM e as demais manifestações associadas, seria de se esperar que providências fossem tomadas no sentido de apoio e assistência psicoterapêutica ao estudante. 


\section{Serviços de apoio acadêmico e psicológico ao aluno em universidades}

Se por um lado, as pesquisas citadas acima revelam uma significativa incidência de TMM e consequente sofrimento psíquico, por outro lado, evidencia-se, pelo menos na literatura nacional, a escassez de estudos sobre serviços de atendimento psicológico a estudantes universitários.

Em Cerchiari, Caetano e Faccenda (2005b), encontramos que esse tipo de serviço teria surgido nos Estados Unidos no início do século XX, a partir do reconhecimento de que os universitários passam por uma fase vulnerável, do ponto de vista psicológico, e que, dessa forma, a responsabilidade em ajudá-los, nesse momento, seria da instituição em que se encontrem inseridos. Este reconhecimento levou à Primeira Conferência Internacional sobre Saúde Mental Estudantil, da qual participaram 37 especialistas de diferentes categorias profissionais, oriundos de vários países. Já no que concerne ao Brasil, os autores situam como marco inicial do atendimento em saúde mental a universitários, a criação, no ano de 1957, na Faculdade de Medicina da Universidade Federal de Pernambuco, do primeiro Serviço de Higiene Mental e Psicologia Clínica, com a finalidade de oferecer assistência psicológica e psiquiátrica aos estudantes universitários.

Figueiredo e Oliveira (1995) apresentam a tentativa de implantação de um serviço dessa natureza junto ao corpo discente da Universidade Federal de São Carlos (UFSCar). Foi realizado um estudo prévio com os estudantes, no qual foi possível constatar que os interesses se concentravam em: problemas emocionais $(37 \%)$, doenças sexualmente transmissíveis (24\%), toxicomania $(17,4 \%)$, métodos anticoncepcionais $(10,7 \%)$ e alcoolismo $(2,6 \%)$.

Cianflone, Figueiredo e Colares (2002) apresentam o Centro de Apoio Educacional e Psicológico (CAEP), que tem como finalidade a assistência psicológica e psicopedagógica aos alunos de graduação da Faculdade de Medicina de Ribeirão Preto da Universidade de São Paulo. O objetivo geral desse serviço é auxiliar o estudante no seu desenvolvimento pessoal e profissional, no decorrer de sua formação acadêmica. As modalidades de atendimento são variáveis e procuram se adaptar à disponibilidade dos alunos e às possibilidades do serviço.

Peres, Santos e Coelho (2003) descrevem em seu trabalho o Programa de Pronto-Atendimento Psicológico ao Aluno, um serviço de atendimento psicológico oferecido junto à clínica-escola da Faculdade de Ciências e Letras de Assis da Unesp e o Centro de Pesquisas e Psicologia Aplicada, voltado à comunidade discente dessa universidade. Segundo a descrição feita, o serviço oferece atendimentos gratuitos aos estudantes, conduzidos por estagiários dos $4^{\circ}$ e $5^{\circ}$ anos do curso de graduação em Psicologia e supervisionados por uma psicóloga contratada.

Assis e Oliveira (2010) apresentam um levantamento realizado pelo Fórum de Pró-reitores de Assuntos Comunitários e Estudantis - órgão ligado à Associação Nacional de Reitores das Universidades Federais Brasileiras. Este fórum realizou um mapeamento sobre a assistência aos estudantes praticada entre 40 IES públicas brasileiras, durante os anos de 1999 e 2000. Dessas 40 instituições, eles constataram que somente $34 \%$ ofereciam algum tipo de atendimento à saúde mental dos estudantes universitários.

Bisinoto e Marinho-Araújo (2015) realizaram estudo pioneiro que mapeou os Serviços de Psicologia das Instituições de Educação Superior brasileiras e o trabalho realizado pelos psicólogos escolares, por meio de questionário (formulário) eletrônico, composto de 13 questões objetivas, tendo identificado 87 Serviços. Verificaram que, na maioria deles, os psicólogos escolares, em equipe multiprofissional, desenvolvem ações principalmente direcionadas aos estudantes, identificando-se algumas intervenções com outros atores educacionais. Em outro texto, Bisinoto e Marinho-Araújo (2014) apresentam uma proposta de atuação para os Serviços de Psicologia Escolar na Educação Superior, abordando diversos aspectos destes serviços como: objetivos, público-alvo, recursos humanos e as atividades a serem desenvolvidas.

Em conclusão, o que se verifica, em relação ao atendimento psicoterapêutico ao estudante universitário, em geral, e aos dos cursos de Psicologia, em particular, é que a situação ainda demanda mais investigações. Mas, pelos dados que já se possui, verifica-se uma oferta muito aquém da demanda por este tipo de atendimento, levando à reivindicação de ações dos órgãos responsáveis pela fiscalização da formação e do exercício profissional, no sentido de melhoria de tão indispensável dispositivo de saúde universitária.

\section{Perspectiva do estudo}

A partir do panorama da literatura científica apresentado anteriormente, assumiu-se investi- 
gar as vivências acadêmicas dos alunos do curso de Psicologia de um município do interior paulista. Vivência acadêmica é um termo que se tem empregado na literatura de Psicologia aplicada ao ensino superior. Usualmente refere-se a um conjunto geral de aspectos de experiências universitárias, os quais se refletem no desempenho e sucesso dos alunos. Ao utilizar este termo autores procuram extrapolar a tradicional perspectiva intrapsíquica e contemplar também características dos contextos universitários, ou seja, fatores externos ao indivíduo. Almeida, Soares e Ferreira (2000) destacam como relacionados a essa vivência os domínios: acadêmico, social, pessoal e vocacional. Subdividido em dimensões e escalas, este constructo foi avaliado na forma de questionário, construído e validado em Portugal (Almeida, 1998) e adaptado ao Brasil (Granado, Santos, Almeida, Soares, \& Guisande, 2005).

Entre os poucos estudos empíricos encontrados na literatura e que investigam aspectos das vivências acadêmicas em curso de Psicologia temos o de Igue, Barani e Milanesi (2008), que pesquisaram as vivências acadêmicas de universitários por meio da aplicação do Questionário de Vivências Acadêmicas - versão reduzida (QVA-r) juntamente com uma questão sobre as expectativas no período de ingresso à universidade em 203 estudantes dos $1^{\circ}$ e $5^{\circ}$ anos de um curso superior de Psicologia. As análises estatísticas indicaram diferença significativa na dimensão institucional entre os dois anos considerados. Quanto às expectativas iniciais, foi encontrada diferença significativa apenas para os alunos de $5^{\circ}$ ano, na dimensão interpessoal e no total das dimensões.

Santos, Polydoro, Scortegagna e Linden (2013), utilizando o QVA-r e uma Escala de Satisfação Acadêmica (ESEA), com 203 estudantes dos cursos de Psicologia $(n=76)$ e Odontologia $(n=127)$ de uma universidade particular, mostraram que os estudantes apresentaram níveis de integração e de satisfação superior à média. Foram detectadas diferenças entre os cursos relativas às variáveis focalizadas e correlações entre as dimensões. Em conclusão, afirmam que a integração e a satisfação, apesar de compartilhar aspectos comuns, avaliam construtos diferentes, ambos importantes para a compreensão do fenômeno complexo e multideterminado da vida acadêmica.

Mais especificamente, investigando reflexões derivadas da experiência como professora e supervisora da disciplina de Técnicas de Diagnóstico Infan- til, a qual exige a realização de um psicodiagnóstico, Poletto (2003) realizou o levantamento e estudo sobre as diversas demandas envolvidas no processo, por meio de exemplos vivenciados pelas alunas com as crianças e seus pais. Na Universidade do Porto, Rocha, Silva, Barbosa e Duarte (2013) buscaram promover o Desenvolvimento Profissional (DP) de estudantes de Psicologia, em duas componentes (intrapessoal e interpessoal/social), por meio de um programa, com dois grupos (um de 10 e outro de cinco participantes), avaliados formalmente no início e no fim do programa, através de uma medida construída para o efeito. Os resultados apoiaram a eficácia da intervenção e indicam mudanças ao nível do autoconhecimento, da representação de DP, do conhecimento do papel de psicólogo, dos recursos para a transição entre papéis e contextos, da coerência e riqueza narrativa e das competências relacionais básicas.

Para a pesquisa que se relatará a seguir, elaborou-se um questionário cuja finalidade era compreender alguns dos fatores mais relevantes que podem levar ao sofrimento psíquico, às dificuldades de envolvimento dos alunos no curso e à evasão, para tanto foi realizado um levantamento acerca das vivências acadêmicas dos alunos do curso de Psicologia de uma universidade pública do interior paulista. Como objetivos específicos, a pesquisa pretendeu investigar respostas dos alunos sobre: como se dava o envolvimento deles com as atividades curriculares, acadêmicas complementares e extracurriculares; os relacionamentos interpessoais com colegas e professores; as justificativas para o trancamento geral/evasão do curso; e possíveis indícios de sofrimento psíquico.

\section{Método}

\section{Caracterização do estudo}

Trata-se de uma pesquisa transversal de abordagem qualitativa e quantitativa realizada por meio de questionário. $\mathrm{O}$ questionário utilizado continha sete questões: a primeira delas de caracterização sociodemográfica, a segunda uma escala Likert, as outras cinco eram questões abertas, as quais foram respondidas anonimamente pelos estudantes do curso de Psicologia de uma universidade pública do interior do estado de São Paulo. 


\section{Caracterização dos participantes}

Participaram deste estudo 119 discentes matriculados do $1^{\circ}$ ao $5^{\circ}$ ano do curso de Psicologia da referida IES, que oferece 40 vagas anuais aos ingressantes, podendo totalizar até 200 alunos. Foram incluídos todos os alunos que, estando presentes no momento da aplicação do questionário, concordaram em participarem. Destes, $32 \%$ possuíam idade de $21-22$ anos, $28 \%$, de $19-20$ anos, $24 \%$, de $23-24$ anos; $61 \%$ eram do sexo feminino; $89 \%$ não exerciam atividade remunerada; $48 \%$ declararam morar com colegas; $61 \%$ declararam que a mãe possui ensino superior completo e $56 \%$, que o pai possui ensino superior completo.

\section{Instrumento utilizado}

Foi elaborado um questionário com quatro partes. A primeira parte solicitava dados sociodemográficos dos alunos, tais como idade, gênero, estado civil, escolaridade e profissão dos pais, entre outros. A segunda parte tratava-se de uma Escala Likert de cinco pontos (1 - Discordo muito; 2 - Discordo; 3 - Indiferente; 4 - Concordo; 5 - Concordo plenamente), elaborada com 20 itens, dos quais 10 foram aproveitados do "Questionário de Vivências Acadêmicas revisto - QVA-r” (Almeida Soares \& Ferreira, 2002), sendo que das cinco dimensões que o compõe, não foi utilizado nenhum item da Dimensão Pessoal, 1 da Dimensão Vocacional, 2 da Dimensão Interpessoal, 3 da Dimensão Estudo-Aprendizagem e 4 da Dimensão Institucional. Exemplos: "Sinto-me envolvido com o meu curso", "Mesmo que pudesse não mudaria de curso", "Conheço bem os serviços oferecidos pela universidade", "Simpatizo com a cidade onde se situa a minha universidade". Estes itens foram completados com outros 10 , das quais cinco se referiam diretamente a aspectos do curso (carga horária, atividades de sala de aula, atividades práticas, tipos de avaliações e desempenho nas provas), três se referiam às atividades acadêmicas complementares (iniciação científica, monitoria e extensão universitária), um sobre atividades extracurriculares e ainda outro sobre as relações das unidades de conteúdos do início do curso com as do final (A Tabela 1, na seção de Resultados, apresenta os enunciados dos 20 itens). A terceira parte era composta por três itens de múltipla escolha, com opções: "sim", "não" ou "não sei”, sendo a primeira questão sobre "espaço para discussão das dificuldades de adaptação no curso", a segunda sobre "a importância da discussão de questões relativas ao papel profissional do psicólogo no início do curso", a terceira sobre "trancamento total de matrícula". Esta última questão subdivide a resposta "sim" em três alternativas considerando-se o número de semestres trancados. Além disso, este item solicita que o respondente declare os motivos que levaram ao trancamento total. A quarta parte do questionário compreendia duas questões abertas, a primeira delas sobre "vivências acadêmicas" e a segunda sobre "sofrimento psíquico".

\section{Procedimento}

Próximo ao final do 2o semestre letivo, no início ou término das aulas, com a anuência dos docentes, os pesquisadores apresentavam os objetivos da pesquisa, descreviam seus procedimentos e convidavam os estudantes presentes em sala a participarem do estudo. Os que concordavam assinavam o Termo de Consentimento Livre e Esclarecido e respondiam o questionário. Foi escolhido o final de semestre por se considerar que a vivência acumulada durante o ano iria possibilitar, especialmente aos ingressantes, condições para responderem às questões propostas. Foi dada preferência à aplicação no início ou término de disciplinas obrigatórias, por concentrarem maior número de estudantes. Pelo mesmo motivo se preferiu a aplicação em sala de aula.

\section{Análise de dados}

Nas primeira, segunda e terceira partes, os dados foram analisados a partir do cômputo das porcentagens de resposta em cada uma de suas alternativas, sejam as questões de múltipla escolha ou as da Escala Likert. Para esta, foram calculados os Box Scores e a Moda de cada item (segundo as recomendações de Jamieson, 2004). Três itens da Escala apresentavam formulações negativas, portanto para melhor visualização tiveram a sua pontuação invertida. Para toda a Escala Likert foi calculado o intervalo de confiança de proporções do Top e Botton Box totais, com a finalidade de estimar quais seriam as proporções de respostas extremas se todos os alunos estivessem presentes durante a aplicação (Jamieson, 2004). As respostas dadas na Quarta parte do questionário, de questões abertas, foram submetidas à análise de conteúdo. 


\section{Considerações éticas}

A pesquisa foi aprovada pelo comitê de ética em pesquisa com seres humanos da IES a qual pertencem os autores (CAAE no 22877113.4.0000.5407).

\section{Resultados}

A seguir, serão apresentados os resultados obtidos na análise dos dados da segunda (Questão II), terceira (Questões III, IV e V) e quarta (Questões VI e VII) partes do questionário aplicado aos estudantes, uma vez que os dados da primeira parte já foram incluídos na caracterização dos participantes na seção de Método.

\section{Vivências acadêmicas avaliadas pela Escala Likert (Questão II)}

A Tabela 1 apresenta as respostas brutas aos 20 itens que compuseram a Escala Likert (Questão II), nas colunas estão: o enunciado da questão; as frequências brutas de respostas em cada um dos pontos da escala de 1 a 5 (as modas destacadas em negrito); o Botton Box (porcentagem de respostas no extremo

Tabela 1

Frequência bruta de respostas a cada ponto e em cada item da Escala Likert, com modas destacadas em negrito e Botton e Top Box por item.

\begin{tabular}{|c|c|c|c|c|c|c|c|}
\hline \multirow[t]{2}{*}{ Enunciado } & \multicolumn{5}{|c|}{$\begin{array}{l}\text { Frequências brutas em } \\
\text { cada um dos pontos da } \\
\text { escala }\end{array}$} & \multirow{2}{*}{$\begin{array}{l}\text { Bottom Box } \\
\text { [Muito ruim/ } \\
\text { Discordo } \\
\text { muito (1)] }\end{array}$} & \multirow{2}{*}{$\begin{array}{c}\text { Top Box } \\
\text { [Muito boa/ } \\
\text { Concordo } \\
\text { plenamente (5)] }\end{array}$} \\
\hline & 1 & 2 & 3 & 4 & 5 & & \\
\hline $\begin{array}{l}\text { Gostaria de concluir o curso na instituição } \\
\text { que frequento }\end{array}$ & 2 & 0 & 5 & 16 & 96 & $1,68 \%$ & $82,04 \%$ \\
\hline Mesmo que pudesse não mudaria de curso & 12 & 9 & 6 & 25 & 67 & $10,08 \%$ & $62,56 \%$ \\
\hline $\begin{array}{l}\text { A instituição de ensino que frequento não me } \\
\text { desperta interesse* }\end{array}$ & 2 & 4 & 11 & 39 & 63 & $1,68 \%$ & $53,84 \%$ \\
\hline $\begin{array}{l}\text { Oportunidades de atividades de pesquisa } \\
\text { (IC e outros) }\end{array}$ & 0 & 2 & 14 & 44 & 59 & $0 \%$ & $49,58 \%$ \\
\hline $\begin{array}{l}\text { Simpatizo com a cidade onde se situa minha } \\
\text { universidade }\end{array}$ & 4 & 5 & 11 & 42 & 57 & $3,36 \%$ & $49,55 \%$ \\
\hline Relacionamento interpessoal com colegas & 2 & 3 & 12 & 48 & 54 & $1,68 \%$ & $46,15 \%$ \\
\hline Tenho dúvidas vocacionais* & 10 & 13 & 15 & 37 & 44 & $8,40 \%$ & $40,34 \%$ \\
\hline Sinto-me desiludido(a) com meu curso* & 5 & 13 & 12 & 48 & 41 & $4,20 \%$ & $35,95 \%$ \\
\hline Sinto-me envolvido com o meu curso & 3 & 10 & 19 & 48 & 39 & $2,52 \%$ & $33,61 \%$ \\
\hline $\begin{array}{l}\text { Oportunidades de atividades de extensão } \\
\text { universitária }\end{array}$ & 2 & 31 & 34 & 26 & 26 & $1,68 \%$ & $22,22 \%$ \\
\hline Relacionamento interpessoal com docentes & 3 & 11 & 43 & 44 & 18 & $2,52 \%$ & $15,51 \%$ \\
\hline Desempenho nas provas & 0 & 5 & 25 & 72 & 17 & $0 \%$ & $14,29 \%$ \\
\hline $\begin{array}{l}\text { Envolvimento em atividades } \\
\text { extracurriculares }\end{array}$ & 7 & 25 & 34 & 38 & 15 & $5,88 \%$ & $13,39 \%$ \\
\hline Carga horária semanal de atividades do curso & 2 & 33 & 35 & 39 & 10 & $1,68 \%$ & $8,55 \%$ \\
\hline Atividades práticas & 8 & 31 & 36 & 35 & 9 & $6,72 \%$ & $8,10 \%$ \\
\hline Quantidade e qualidade das monitorias & 4 & 27 & 45 & 36 & 7 & $3,36 \%$ & $6,09 \%$ \\
\hline Nível de exigências das avaliações & 4 & 16 & 40 & 54 & 5 & $3,36 \%$ & $4,35 \%$ \\
\hline $\begin{array}{l}\text { Conheço bem os serviços oferecidos pela } \\
\text { universidade }\end{array}$ & 5 & 36 & 41 & 32 & 5 & $4,20 \%$ & $4,38 \%$ \\
\hline $\begin{array}{l}\text { Relações entre as disciplinas iniciais e a } \\
\text { prática profissional }\end{array}$ & 18 & 32 & 46 & 19 & 4 & $15,13 \%$ & $3,95 \%$ \\
\hline Atividades em sala de aula & 0 & 16 & 55 & 46 & 2 & $0 \%$ & $1,68 \%$ \\
\hline Total & & & & & & $3,91 \%$ & $27,81 \%$ \\
\hline
\end{tabular}

IC: Iniciação científica.

* Itens cujas pontuações foram invertidas devido ao sentido negativo da questão. 
inferior da escala [ponto 1]) e o Top Box (porcentagem no extremo superior [ponto 5]).

Nos seis primeiros itens da Tabela 1, a moda se situa na pontuação 5 e há uma diferença de mais de 44 pontos percentuais entre seus respectivos Botton Box e Top Box. Os estudantes têm uma percepção muito positiva das questões abordadas nos respectivos itens: opinião sobre a instituição e o curso; oportunidades de pesquisa; a cidade da universidade; e relacionamento interpessoal com colegas.

A moda do sétimo item ("Tenho dúvidas vocacionais") se situa na pontuação 5 e a diferença entre $T o p$ e Botton Box é superior a $30 \%$, mas $8,4 \%$ das respostas foram na pontuação mais negativa do item. Há um número considerável de alunos com dúvida vocacional.

Para os cinco itens subsequentes, a moda está nas pontuações 4 ou 3, com a diferença entre o Top e Botton Box variando de 13 a 31 pontos percentuais. Os estudantes que de maneira geral se sentem envolvidos e satisfeitos com o curso e com seu desempenho neste têm um bom relacionamento interpessoal com docentes e julgam que têm boas oportunidades de atividades de extensão universitária.

A moda dos seis itens a seguir se situa nas pontuações 3 e 4, mas com diferenças entre Top e Botton Box pequenas (menores que 8\%). Os alunos não se sentem muito envolvidos com atividades extracurriculares; julgam que a carga horária semanal do curso não é muito adequada; julgam as atividades práticas como medianas; não concordam muito com a quantidade de monitorias oferecidas e o nível de exigência das avaliações; e não conhecem muito bem os serviços oferecidos pela universidade. O último item ("Atividades em sala de aula") contém somente duas respostas nas categorias extremas. Estas respostas condizem com o fato dos alunos desejarem melhores aulas, mais atividades práticas, melhores avaliações e menor carga horária semanal.

No penúltimo item ("Relações entre as disciplinas iniciais e a prática profissional”), o Botton Box excede consideravelmente o Top Box. A moda se situa na pontuação 3 , mas a concentração de respostas se dá nas três primeiras pontuações de resposta. Os alunos consideram muito ruim a relação entre a teoria ministrada em aula e a prática profissional, em coerência com as respostas aos itens de atividades práticas e atividades em sala de aula.

Em síntese, nos itens da Escala Likert, os alunos apresentaram, no geral, uma apreciação bastante favorável do curso.

\section{Necessidade de reflexão sobre o curso e trancamentos (Questões III, IV e V)}

As questões de múltipla escolha III, IV e V abordaram as necessidades de reflexão, de acolhimento e a evasão do curso. As alternativas eram "Sim", "Não" e "Não sei".

Na Questão III, pergunta-se: "Você considera importante que os alunos tenham um espaço para discussões em grupo, sobre as dificuldades de adaptação ao início do curso?" Essa questão foi respondida afirmativamente por 115 alunos (96,6\%), revelando um consenso dominante entre os alunos sobre a importância de apoio ao aluno no momento inicial do curso.

As respostas à Questão IV ("Você acha importante que se discuta, no início do curso questões relativas ao papel profissional do psicólogo?") mostraram uma concentração maior dos dados, com três turmas (10, 3o e 5o anos) apresentando consenso (100\% de frequência na alternativa "Sim"). No geral, 116 alunos $(97,4 \%)$ responderam afirmativamente à questão, revelando um interesse generalizado em discutir aspectos da atuação profissional, e ele se estende a toda a graduação.

A Questão V buscou detectar quantos alunos haviam passado pelo processo de trancamento total do curso em anos recentes, o que poderia indicar uma tendência à evasão. À exceção do primeiro, um aluno de cada ano havia trancado e retomado o curso, todos por um período de até dois semestres, totalizando quatro alunos (3,4\% das respostas). O restante, 115 estudantes $(96,6 \%)$, nunca realizara trancamento. Para aqueles que responderam afirmativamente a essa questão, solicitou-se os motivos que levaram ao referido trancamento. Como respostas, apareceram a "não identificação com a proposta das disciplinas", "problemas de saúde com membro da família", "carga horária excessiva de comprometimento com atividades" e "motivos pessoais que impossibilitaram a conciliação com atividades do curso". Evidentemente, alunos que estavam com a matrícula trancada no momento de aplicação do questionário não responderam a esta questão. Dados da época da aplicação do questionário mostram que, de quatro turmas ativas naquele período, haviam evadido 17 alunos, aproximadamente quatro em cada turma de 40 , ressalvando-se que estas vagas são sempre preenchidas por transferência. 


\section{Comentários abertos sobre as vivências e o sofrimento psíquico (Questões VI e VII)}

A Análise de Conteúdo das repostas à Questão VI ("Comente a sua vivência acadêmica até o momento, considerando seus relacionamentos, acontecimentos marcantes, facilidades e dificuldades?") resultou em categorias que foram classificadas segundo a valoração que os estudantes atribuíam a estes temas, separando-as em: "favorável", "desfavorável" ou "neutra" (Tabela 2).

Com predomínio de classificação favorável estão as incluídas nas categorias: "referência às vivências em geral” (58,8\% de respostas favoráveis, em 64$)$; o "relacionamento entre pares" $(78,4 \%$ de respostas favoráveis, em 51); "atividades extracurriculares" (94,1\% de respostas favoráveis, em 17); e "relacionamento com os professores" $(48,1 \%$ de respostas favoráveis e 40,8\%, desfavoráveis, em 27 ).

Como desfavoráveis: a "carga horária", com relatos de práticas avaliativas recorrentemente se restringindo a atividades centralizadas apenas nos finais dos semestres, sobrecarregando esse período, enquanto em outros momentos se sentem ociosos; a "organização curricular"; críticas à excessiva con- centração de disciplinas básicas nos primeiros anos; a "relação entre teoria e prática"; e "adaptação à vida universitária". Esta última, com relatos abordando desde situação econômica até a iniciação e cultivo de laços sociais, frente à situação de risco de ruptura de vínculos, mudança de cidade, inserção institucional e até questões relativas à formação de identidade. A dificuldade de acesso a "informações institucionais" de serviços que a universidade já oferece e que não comunica de forma eficiente e a falta de transparência da instituição, que tem suas informações concentradas em um sistema pouco prático, fazendo com que as oportunidades dependam muito de contatos informais para suas identificações.

Em síntese, são diversos os indicadores de situações internas ao curso que podem levar a sentimentos de inadequação e até mesmo a sofrimento. A despeito do posicionamento aparentemente favorável explicitado na escala Likert incluída na Questão II.

A última questão (VII: "Você considera que as atividades desenvolvidas pelos alunos deste curso podem desencadear algum tipo de sofrimento psíquico? Explique"), obteve 107 respostas "sim" (90\%), explicadas posteriormente, e 12 respostas "não" (10\%), sem justificati-

Tabela 2

Frequência e porcentagem das categorias de conteúdos obtidas a partir das respostas à Questão VI.

\begin{tabular}{|c|c|c|c|c|}
\hline Categorias & Favorável & Neutro & Desfavorável & Total \\
\hline Referência às vivências em geral & $37(58,7 \%)$ & $16(25,4 \%)$ & $10(15,9 \%)$ & 63 \\
\hline Relacionamento entre pares & $40(78,4 \%)$ & $2(3,9 \%)$ & $9(17,7 \%)$ & 51 \\
\hline Relacionamento com professores & $13(48,1 \%)$ & $3(11,1 \%)$ & $11(40,8 \%)$ & 27 \\
\hline Carga horária & $2(8,0 \%)$ & 0 & $23(92,0 \%)$ & 25 \\
\hline Adaptação à vida universitária & 0 & $1(4,8 \%)$ & $20(95,2 \%)$ & 21 \\
\hline Questões específicas dos dois primeiros anos & $2(9,5 \%)$ & $1(4,8 \%)$ & $18(85,7)$ & 21 \\
\hline Atividades extracurriculares & $16(94,1 \%)$ & 0 & $1(5,8 \%)$ & 17 \\
\hline Relação entre teoria e prática & $2(12,5 \%)$ & 0 & $14(87,5 \%)$ & 16 \\
\hline Didática & $2(15,4 \%)$ & $1(7,7 \%)$ & $10(76,9 \%)$ & 13 \\
\hline Organização curricular & 0 & $1(7,7 \%)$ & $12(92,3 \%)$ & 13 \\
\hline Informações institucionais & $1(8,3 \%)$ & $2(16,7 \%)$ & $9(75,0 \%)$ & 12 \\
\hline Relacionamento com veteranos & $6(54,5 \%)$ & $1(9,1 \%)$ & $4(36,4 \%)$ & 11 \\
\hline Sociabilização ou eventos sociais & $7(70,0 \%)$ & 0 & $3(30,0 \%)$ & 10 \\
\hline Experiência de estágios & $1(16,7 \%)$ & $3(50,0 \%)$ & $2(33,3 \%)$ & 6 \\
\hline Atividades de pesquisa e extensão & $6(100 \%)$ & 0 & 0 & 6 \\
\hline Informações acadêmicas & $1(20,0 \%)$ & 0 & $4(80,0 \%)$ & 5 \\
\hline Avaliação & 0 & 0 & $3(100 \%)$ & 3 \\
\hline Pressão por desempenho & 0 & 0 & $3(100 \%)$ & 3 \\
\hline Motivação em relação ao curso & 0 & $1(33,3 \%)$ & $2(66,7 \%)$ & 3 \\
\hline Eventos acadêmicos & $3(100 \%)$ & 0 & 0 & 3 \\
\hline Vocação & 0 & 0 & $1(100 \%)$ & 1 \\
\hline
\end{tabular}


vas. A Análise de Conteúdo destas produziram diversas categorias, sendo possível que uma mesma resposta, pela sua extensão, produzisse mais de um sentido.

A "carga excessiva de atividades do curso" é a categoria com maior número de respostas (28), com exigência do aluno de mais tempo do que o previsto no programa das disciplinas, que dificulta a participação em atividades extracurriculares. A "natureza específica do estudo da Psicologia" (27 respostas) inclui referências à mobilização de sentimentos e abordagem de temas de caráter pessoal. A seguir, a "exigência emocional durante os estágios que acontecem nos dois últimos anos do curso" (17 respostas); o "exagero de demanda encontrada na cultura pelo desempenho acadêmico", citado em 16 respostas, "Necessidades de um sistema de apoio ao aluno e sugestões específicas", em 15. Nesta última categoria, os respondentes também abordaram o sucateamento de um órgão já existente que oferece serviço clínico para membros da instituição, assim como a inexistência e a necessidade de sistemas abertos de apoio pessoal ao estudante.

"Relacionamentos com professores específicos" incluiu 13 respostas; "Organização curricular" incluiu 12; "Especificidades estruturais do primeiro ano" incluiu 10. Houve oito repostas que fizeram referência ao surgimento de sintomas psicossomáticos secundários a circunstâncias vivenciadas no curso, entre tais sintomas foram citados: a privação de sono, o estresse e angústias facilitadoras de desequilíbrios hormonais.

Categorias com poucas respostas em cada uma delas: "necessidades ou demandas estudantis não atendidas" com seis respostas; "avaliações sem sentido" com cinco; "dissociação teoria e prática" e "falta de didática", ambas com quatro respostas cada; "dificuldade de adaptação à vida universitária" com três respostas; "dúvidas vocacionais" apenas duas; e por último uma categoria "outras", com as seguintes respostas: "problemas de socialização e interação entre os alunos", "relacionamento com os veteranos", "falta de reconhecimento para as atividades extracurriculares" e "menção a evasão que aconteceu com colega por não ter recebido apoio institucional”.

Em resumo, nestas duas questões (VI e VII) foram reveladas situações de produção de sofrimento psíquico vivenciadas por estudantes até mesmo em um curso de Psicologia que, numa avaliação geral e objetiva, se apresentava como bom.

Numa síntese geral dos resultados evidencia-se, inicialmente, que a percepção do curso pelos alunos é bastante satisfatória. Mas, se deve considerar ser esta apenas uma aproximação bastante geral das vivências do curso em análise, pois foi possível a identificação de alguns aspectos mais específicos nos quais os estudantes apontavam problemas. Por exemplo, o penúltimo item da Escala Likert ("Relações entre as disciplinas iniciais e a prática profissional”) foi o único no qual o número de avaliações extremamente negativas excede consideravelmente o número de avaliações extremamente positivas. Mais explicitamente, na Questão VI, os estudantes fizeram várias referências desfavoráveis à relação entre teoria e prática, didática e organização curricular. E na Questão VII, ao serem perguntados sobre vivências que podem desencadear sofrimento psíquico, os alunos relacionam esse sofrimento a natureza particular do estudo da Psicologia, a exigência emocional durante os estágios que acontecem nos dois últimos anos do curso, organização curricular e especificidades do 10 ano de curso.

Além disso, o perfil traçado pelos alunos caracteriza as disciplinas do curso, em especial as iniciais, como desorganizadas entre si, sem comunicação entre os docentes. Também não fica evidente a relação das disciplinas dos anos iniciais com a prática da profissão, o que pode acarretar em dificuldades no momento em que são solicitados a realizarem os estágios específicos, nos dois últimos anos do curso. Muitas dessas questões poderiam ser trabalhadas com o aluno, se a faculdade promovesse discussões no início do curso sobre questões relativas ao papel profissional do psicólogo. Prática que quase todos os participantes consideraram importante. O perfil é de alunos sobrecarregados. A Questão II mostra a carga horária semanal avaliada como não sendo muito adequada. Nas questões abertas VI e VII, a carga horária é avaliada negativamente também, sendo considerada excessiva em especial nos momentos finais de cada semestre.

\section{Discussão}

Um primeiro aspecto, evidenciado na Escala Likert (Questão II), se refere às dúvidas vocacionais. Este é um aspecto que poderia ser trabalhado com tais alunos em serviços de apoio ao aluno, tais como os citados por Assis e Oliveira (2010), Bisinoto e Marinho-Araújo (2015), Cerchiari et al. (2005b), Cianflone et al. (2002), Figueiredo e Oliveira (1995), Peres et al. (2003). Esta questão talvez não tenha surgido com maior destaque nos dados pelo fato 
de que a coleta de dados foi realizada próxima ao final do 20 semestre, época em que as dúvidas vocacionais maiores já resultaram em evasão, tal como corroborado pelos estudos de Marinho-Araujo e Bisinoto (2011), Reason et al. (2006) e Teixeira et al. (2008), que apontam que os maiores índices de evasão ocorrem ao final do 10 ano.

Informações da IES na qual a pesquisa foi realizada apontam que a evasão naquele curso se situa entre $10 \%$ e $20 \%$, quase sempre ao final do 10 ano. Evidentemente, os dados com os alunos evadidos não poderiam ter sido conseguidos pelo questionário utilizado, pois, pela sua natureza, ele demandava uma vivência mínima com curso, antes de ser respondido, daí a sua aplicação mais próxima ao final do ano, quando os evadidos do 10 ano já não cursavam mais as disciplinas. Como apontado por Marinho-Araujo e Bisinoto (2011), Reason et al. (2006), Teixeira et al. (2008), o 10 ano de faculdade é um momento crítico para os alunos.

Na questão final sobre o sofrimento psíquico que pudessem ter vivenciado, os resultados obtidos apontam uma real existência de dificuldades de adequação ao curso e, principalmente, a ocorrência de sofrimento psíquico apontada por 107 dos 119 respondentes. Na revisão da literatura, apresentada na Introdução, para Cerchiari et al. (2005a) e Neves e Dalgalarrondo (2007), há uma prevalência maior de Transtornos Mentais Menores em estudantes dos cursos de ciências humanas e da saúde. Também Facundes e Ludemir (2005) encontraram um índice geral de $34,1 \%$, com predominância entre estudantes de Medicina (42,6\%). Ainda Fiorotti et al. (2010) e Lima et al. (2006) encontraram índices de $44,6 \%$ e $37,1 \%$, respectivamente, entre os graduandos em medicina. Assim, como Cavestro e Rocha (2006), sobre os índices de depressão e risco de suicídio entre estudantes. Os autores de todos esses estudos observaram que o contato com o sofrimento psíquico de outras pessoas ou os conteúdos acadêmicos mais diretamente relacionados com a subjetividade humana correlaciona-se com certa vulnerabilidade e pode elevar as probabilidades do desenvolvimento de algum transtorno mental, o que leva à hipótese de que o mesmo ocorra com estudantes dos cursos de Psicologia. O que foi corroborado pelos dados obtidos na presente pesquisa.

Todos estes aspectos justificam a preocupação com a oferta de dispositivos de assistência e apoio ao estudante. Assis e Oliveira (2010), Bisinoto e Marinho-Araújo (2015), Cerchiari et al. (2005b), Cianflone et al. (2002), Figueiredo e Oliveira (1995), Peres et al. (2003), mostram que, em relação ao atendimento ao estudante universitário, em geral, mas também em relação aos dos cursos de Psicologia, em particular, a situação de atendimento psicológico ainda é bastante precária, clamando por investigações e, principalmente, por ações dos órgãos responsáveis pela fiscalização da formação e do exercício profissional no sentido de melhoria de tão indispensável dispositivo de saúde universitária.

Por tratar-se de uma universidade pública paulista, esta negligência só pode ser atribuída a uma ênfase exagerada no produtivismo, entendido como forma de avaliação do desempenho docente fundamentado na produção científica, ou seja, artigos em periódicos. Numa política deste tipo, a graduação acaba por se tornar uma preocupação secundária, que fica evidenciada no valor secundário atribuído às atividades de ensino nas avaliações docentes, na quantidade relativamente baixa de incentivos às atividades de graduação quando comparadas com os incentivos à pós-graduação e à pesquisa.

Formar psicólogos num contexto como esse, a despeito do destaque que o curso possa ter, seja por sua alta procura ou por posições elevadas nos rankings nacionais, fica comprometido pela negligência com as vivências acadêmicas e os aspectos passíveis de gerarem sofrimento psíquico, tal como evidenciado pelos dados apresentados.

\section{Considerações finais}

Os resultados obtidos e discutidos na presente pesquisa permitem a conclusão de uma situação bastante complexa em relação ao sofrimento psíquico dos estudantes deste curso de Psicologia. A percepção geral das vivências no curso, investigadas através da Escala Likert, são amplamente favoráveis, mas, nas questões abertas, evidenciam de forma clara e enfática a existência do sofrimento psíquico, mencionando fatos que os propiciam e apontando vivências que lhes foram desfavoráveis. Estes sentimentos podem estar indicando uma situação de risco aumentado para o desenvolvimento de transtornos mentais nessa população de estudantes, o que sugere a necessidade de haver uma atenção mais cuidadosa, por parte da instituição universitária, com a consequente necessidade de 
discutir e propor medidas de manejo deste sofrimento como medida propiciadora de bem-estar no ambiente universitário.

A partir daí, surge, como aspecto relevante, as propostas de serviços de atendimento ao estudante universitário do curso de Psicologia, modalidade de serviço ainda bastante incipiente, conforme relatado pelos estudos apontados anteriormente. Neste sentido, o Sistema Nacional de Avaliação do Ensino Superior (Sinaes), desenvolvido pelo Instituto Nacional de Estudos e Pesquisas (INEP) do Ministério da Educação (MEC), inclui entre suas dimensões do instrumento de avaliação uma que se refere especificamente à Assistência ao Discente. Esta preocupação do MEC, por certo, determina ações e preocupações em universidades públicas federais, mas não se estende às públicas estaduais que estejam subordinadas a Conselhos Estaduais de Educação. Nestas, o descuido é proeminente, mais ainda nesta época de crise da universidade púbica, como mencionado na Introdução. Na IES do curso no qual a pesquisa se realizou havia um projeto de criação de um Serviço de Atendimento ao Aluno

\section{Referências}

Almeida, L. S. (1998). Questionário de vivências académicas para jovens universitários: estudos de construção e de validação. Revista Galego-Portuguesa de Psicología e Educación, 2(3), 113-130.

Almeida, L. S., Soares, A. P., \& Ferreira, J. A. G. (2000). Transição e adaptação à universidade: apresentação do questionário de vivências académicas (QVA). Psicologia, 14(2), 189-208. doi:10.17575/rpsicol.v14i2.510

Almeida, L. S., Soares, A. P. C., \& Ferreira, J. A. (2002). Questionário de Vivências Acadêmicas (QVA-r): avaliação do ajustamento dos estudantes universitários. Avaliação Psicológica, 1(2): 81-93. Recuperado de: http://pepsic.bvsalud.org/scielo.php?script=sci_arttext\&pid=S1677-04712002000200002

Andrade, J. B. C., Sampaio, J. J. C., Farias, L. M., Melo, L. P., Souza, D. P., Mendonça, A. L.B. et al. (2014). Contexto de formação e sofrimento psíquico de estudantes de medicina. Revista Brasileira de Educação Médica, 38(2), 231-242. doi:10.1590/S0100-55022014000200010 que foi completamente abandonado tão logo a crise se instalou.

Seria, portanto, de se esperar que o Conselho Federal de Psicologia também se ocupasse da mesma. Cabe, pois, propor que, a partir da literatura revista $\mathrm{e}$ dos dados obtidos na presente pesquisa, este órgão assuma ações efetivas seja no sentido de orientar ou até mesmo de fiscalizar a implantação de Serviços de Atendimento ao Estudante de Psicologia.

A pesquisa apresentada neste artigo possui limitações, por utilizar um questionário de autorrelato que revelou dados que podem não refletir de forma completa e aprofundada as percepções que os estudantes possuíam, ainda que se tenha incluído questões abertas. A realização de estudos utilizando, por exemplo, entrevistas poderiam enriquecer os dados obtidos e revelar aspectos que não foram mencionados. Ainda que, pelo compromisso ético assumido, não se mencionou qual foi a IES estudada, fica evidente que se trata de um estudo cujos dados, por princípio, se restringem ao seu caso, estimulando, pois, que novas pesquisas com diversas outras IES públicas ou particulares se realizem.

Assis, A. D., \& Oliveira, A. G. B. (2010). Vida universitária e saúde mental: Atendimento às demandas de saúde e saúde mental de estudantes de uma universidade brasileira. Cadernos Brasileiros de Saúde Mental, 2(4-5), 159-177. Recuperado de: http:// incubadora.periodicos.ufsc.br/index.php/cbsm/ article/view/1113/1305

Bardagi, M. P., \& Hutz, C. S. (2009). "Não havia outra saída”: percepções de alunos evadidos sobre o abandono do curso superior. PsicoUSF, 14(1), 95-105. doi:10.1590/S1413-82712009000100010

Bisinoto, C., \& Marinho-Araújo, C. M. (2015). Psicologia escolar na educação superior: panorama da atuação no Brasil. Arquivos Brasileiro de Psicologia, 67(2), 33-46. Recuperado de http://pepsic. bvsalud.org/scielo.php?script=sci_arttext\&pi$\mathrm{d}=$ S1809-52672015000200004

Bisinoto, C., \& Marinho-Araújo, C. M. (2014). Serviços de psicologia escolar na educação superior: uma proposta de atuação. In R. Guzzo (Org.), Bastidores da escola e desafios da educação pública: a pesquisa e a prática em psicologia escolar (pp. 277-296). Campinas, SP: Alínea. 
Brasil. Ministério da Educação. (2015). Conselho Nacioal de Educação; Câmara de Educação Superior. Resolução no 5 , de 15 de março de 2011. Institui as Diretrizes Curriculares Nacionais para os cursos de graduação em Psicologia, estabelecendo normas para o projeto pedagógico complementar para a Formação de Professores de Psicologia.Recuperado de http:// portal.mec.gov.br/index.php?option=com_docman\&view=download\&alias=7692-rces005-11-pdf\&category_slug=marco-2011-pdf\&Itemid=30192

Castro, A. K. S. S. (2012). Evasão no ensino superior: um estudo no curso de psicologia da UFRGS. (Dissertação de Mestrado). Universidade Federal do Rio Grande do Sul, Porto Alegre. Recuperado de http:/ / hdl.handle.net/10183/55077

Cavestro, J. M., \& Rocha, F. L. (2006). Prevalência de depressão entre estudantes universitários. Jornal Brasileiro de Psiquiatria, 55(4), 264-267. doi:10.1590/S0047-20852006000400001

Cerchiari, E. A. N., Caetano, D., \& Faccenda, O. (2005a). Prevalência de transtornos mentais menores em estudantes universitários. Estudos de Psicologia, 10(3), 413-420. doi:10.1590/S1413-294X2005000300010

Cerchiari, E. A. N., Caetano, D., \& Faccenda, O. (2005b). Utilizaçãodoserviçodesaúdemental emumauniversidade pública. Psicologia, Ciência e Profissão, 25(2), 252-265. doi:10.1590/S1414-98932005000200008

Chiapetti, H., \& Serbena, C. A. (2007). Uso de álcool, tabaco e drogas por estudantes da área de saúde de uma universidade de Curitiba. Psicologia: Reflexão e Crítica, 20(2),303-313.doi:10.1590/S0102-79722007000200017

Cianflone,A.R.L.,Figueiredo,J.F.C.,\&Colares,M.F.A.(2002). O Centro de Apoio Educacional e Psicológico (CAEP) da Faculdade de Medicina de Ribeirão Preto (USP): história e perspectivas. Medicina (Ribeirão Preto), 35(3), 392396. doi:10.11606/issn.2176-7262.v35i3p392-396

Costa, E. F. O., Rocha, M. M.V., Santos, A. T. R. A., Melo, E.V., Martins, L.A.N., \&Andrade, T.M. (2014).Commonmental disorders and associated factors among final-year healthcare students. Revista da Associação Médica Brasileira, 60(6), 525-530. doi:10.1590/1806-9282.60.06.009

Costa, E. F. O., Andrade, T. M., Silvany Neto, A. M., Melo, E. V., Rosa, A. C. A., Alencar, M. A., \& Silva, A. M. (2010). Common mental disorders among medical students at Universidade Federal de Sergipe: a cross-sectional study. Revista Brasileira de Psiquiatria, 32(1), 11-19. doi:10.1590/S1516-44462010000100005
Facundes V. L. D., \& Ludermir A. B. (2005). Common mental disorders among health care students. Revista Brasileira de Psiquiatria, 27(3), 194-200. doi:10.1590/S1516-44462005000300007

Ferri-de-Barros, J. E., Alencar, M. J., Berchielli, L. F, \& Castelhano Junior, L. C. (2011). Headache among medical and psychologystudents. Arquivosdeneuro-psiquiatria, 69(3), 502-508. doi:10.1590/S0004-282X2011000400018

Figueiredo, R. M., \& Oliveira, M. A. P. (1995). Necessidades de estudantes universitários para implantação de um serviço de orientação e educação em saúde mental. Revista Latino-Americana de Enfermagem, 3(1), 5-18. doi:10.1590/S0104-11691995000100002

Fiorotti, K.P., Rossoni, R. R., Borges, L. H., \& Miranda, A. E. (2010). Transtornos mentais comuns entre os estudantes do curso de medicina: prevalência e fatores associados. Jornal Brasileiro de Psiquiatria, 59(1), 17-23. doi:10.1590/S0047-20852010000100003

Granado, J. I. F., Santos, A. A. A., Almeida, L. S., Soares, A. P., \& Guisande, M. A. (2005). Integração acadêmica de estudantes universitários: contribuitos para a adaptação e validação do QVA-r no Brasil. Psicologia e Educação, 4(2), 31-41.

Horta, R. L., Horta, B. L., \& Horta, C. L. (2012). Uso de drogas e sofrimento psíquico numa universidade do Sul do Brasil. Psicologia em Revista, 18(2), 264-276. doi:10.5752/P.1678-9563.2012v18n2p264

Igue, E. A., Bariani, I. C. D., \& Milanesi, P.V. B. (2008).Vivência acadêmica e expectativas de universitários ingressantes e concluintes. Psico-USF, 13(2), 155-164. doi:10.1590/S1413-82712008000200003

Jamieson, S. (2004). Likert scales: how to (ab) use them. Medical Education, 38(12), 1217-1218. doi:10.1111/j.1365-2929.2004.02012.x

Lima, M.C.P., Domingues, M. S., \& Cerqueira, A. T. A. R. (2006). Prevalência e fatores de risco para transtornos mentais comuns entre estudantes de medicina. Revista de Saúde Pública, 40(6), 1035-41. doi:10.1590/S0034-89102006000700011

Marinho-Araujo, C. M., \& Bisinoto, C. (2011). Psicologia escolar na educação superior. In R. S. L. Guzzo, \& C. M. Marinho-Araujo (Org.), Psicologia escolar: identificando e superando barreiras (pp. 191-214). Campinas, SP: Alínea.

Neves, M. C. C., \& Dalgalarrondo, P. (2007). Transtornos mentais auto-referidos em estudantes universitários. Jornal Brasileiro de Psiquiatria, 56(4), 237-244. doi:10.1590/S0047-20852007000400001 
Peres, R. S., Santos, M. A., \& Coelho, H. M. B. (2003). Atendimento psicológico a estudantes universitários: considerações acerca de uma experiência em clínica-escola. Estudos de Psicologia (Campinas), 20(3),47-57.doi:10.1590/S0103-166X2003000300004

Poletto, R. C. (2003). Demandas do processo psicodiagnóstico: considerações teóricas e clínicas sobre as vivências das estudantes de psicologia. Psicologia: Ciência e Profissão, 23(3), 2-9. doi:10.1590/S1414-98932003000300002

Reason, R. D., Terenzini, P. T., \& Domingo, R. J. (2006). First things first: developing academic competence in the first year of college. Research in Higher Education, 47(2), 149-175. doi:10.1007/s11162-005-8884-4

Rocha, A. C., Silva, G., Barbosa, R., \& Duarte, C. (2013). Tornar-se psicólogo para além das aulas: grupo de desenvolvimento com estudantes de psicologia. Análise Psicológica, 31(1), 87-102. doi:10.14417/ap.660

Santos, A. A. A. G., Polydoro, S. A. J., Scortegagna, S. A., \& Linden, M. S. (2013). Integração ao ensino superior e satisfação acadêmica em universitários. Psicologia: Ciência e Profissão, 33(4), 780-793. doi:10.1590/S1414-98932013000400002

Santos, M. V. R., Pereira, D. S., \& Siqueira, M. M. (2013). Uso de álcool e tabaco entre estudantes de Psicologia da Universidade Federal do Espírito Santo. Jornal Brasileiro de Psiquiatria, 62(1), 22-30. doi:10.1590/S0047-20852013000100004

Silva,A.G.,Cerqueira,A.T.A.R.,\&Lima,M.C.P.(2014).Apoio social e transtorno mental comum entre estudantes de medicina. Revista Brasileira de Epidemiologia, 17(1), 229-242. doi:10.1590/1415-790X201400010018ENG

Silva Filho, R. L. L., Motejunas, P. R., Hipólito, O., \& Lobo, M. B. C. M. (2007). A evasão no ensino superior brasileiro. Cadernos de Pesquisa, 37(132), 641-659. Recuperado de http://publicacoes.fcc.org. br/ojs/index.php/cp/article/view/346/350

Teixeira, M. A. P., Dias, A. C. G., Wottrich, S. H., \& Oliveira, A. M. (2008). Adaptação à universidade em jovens calouros. Psicologia Escolar e Educacional, 12(1), 185-202. doi:10.1590/S1413-85572008000100013

Xavier, A., Nunes, A. I. B. L., \& Santos, M. S. (2008). Subjetividade e sofrimento psíquico na formação do sujeito na universidade. Revista Mal-estar e Subjetividade, 8(2), 427-451. Recuperado de http://pepsic.bvsalud.org/scielo.php?script=sci_arttext\&pi$\mathrm{d}=$ S1518-61482008000200008

\section{Antonio dos Santos Andrade}

Docente da Universidade de São Paulo, Ribeirão Preto - SP. Brasil.

E-mail: antandras@ffclrp.usp.br

Gabriel Arantes Tiraboschi

Mestrando pela Universidade de São Paulo, Ribeirão Preto - SP. Brasil.

E-mail: gabrielarantest@usp.br

\section{Natália Amaral Antunes}

Acadêmica de Psicologia pela Universidade de São Paulo, Ribeirão Preto - SP. Brasil.

E-mail: antunes.poli@gmail.com

\section{Paulo Vinícius Bachette Alves Viana}

Acadêmico de Psicologia pela Universidade de São Paulo, Ribeirão Preto - SP. Brasil.

E-mail:paulobac1@gmail.com

\section{Pedro Alves Zanoto}

Acadêmico de Psicologia pela Universidade de São Paulo, Ribeirão Preto - SP. Brasil.

E-mail: zanoto92@gmail.com

\section{Rafael Trebi Curilla}

Acadêmico de Psicologia pela Universidade de São Paulo, Ribeirão Preto, SP, Brasil

E-mail:wedchidna@hotmail.com

Endereço para envio de correspondência: Faculdade de Filosofia, Ciências e Letras de Ribeirão Preto - USP, Av Bandeiranes, 3900, Ribeirão Preto - São Paulo, CEP: 14049-900

Recebido 29/11/2015

Reformulação 09/08/2016

Aprovado 04/11/2016

Received $11 / 29 / 2015$

Reformulated 08/09/2016

Approved 11/04/2016

Recibido 29/11/2015

Reformulado 09/08/2016

Aceptado 04/11/2016 
Psicologia: Ciência e Profissão Out/Dez. 2016 v. 36 n4, 831-846.

Como citar: Andrade A. S.; Tiraboschi G. A.; Antunes N. A.; Viana P. V. B. A.; Zanoto P. A.; Curilla R. T. (2016). Vivências Acadêmicas e Sofrimento Psíquico de Estudantes de Psicologia. Psicologia: Ciência e Profissão, 36(4): 831-846. DOI: 10.1590/1982-3703004142015

How to cite: Andrade A. S.; Tiraboschi G. A.; Antunes N. A.; Viana P. V. B. A.; Zanoto P. A.; Curilla R. T. (2016). Academic Experiences and Psychological Suffering among Psychology students. Psicologia: Ciência e Profissão, 36(4): página-página.831-846. DOI: 10.1590/1982-3703004142015

Cómo citar: Andrade A. S.; Tiraboschi G. A.; Antunes N. A.; Viana P. V. B. A.; Zanoto P. A.; Curilla R. T. (2016). Vivencias Académicas y el Sufrimiento Psíquico de los estudiantes de Psicología. Psicologia: Ciência e Profissão, 36(4): 831-846. DOI: 10.1590/1982-3703004142015 


\section{ERRATA}

Na edição 36.4, no artigo "Vivências Acadêmicas e Sofrimento Psíquico de Estudantes de Psicologia", nas páginas 831 e 845, onde se lê "Pedro Alvez Zanoto”, correto é: "Pedro Alves Zanoto". 